\title{
An investigation of silicon carbide-water nanofluid for heat transfer applications
}

Cite as: J. Appl. Phys. 105, 064306 (2009); https://doi.org/10.1063/1.3082094

Submitted: 19 November 2008 . Accepted: 10 January 2009. Published Online: 18 March 2009

D. Singh, E. Timofeeva, W. Yu, J. Routbort, D. France, D. Smith, and J. M. Lopez-Cepero

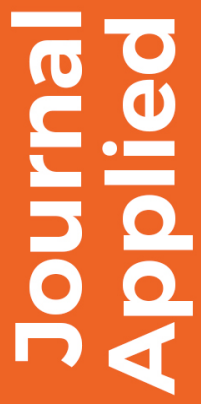

\section{ARTICLES YOU MAY BE INTERESTED IN}

Anomalously increased effective thermal conductivities of ethylene glycol-based nanofluids containing copper nanoparticles

Applied Physics Letters 78, 718 (2001); https://doi.org/10.1063/1.1341218

Base fluid and temperature effects on the heat transfer characteristics of SiC in ethylene glycol/ $\mathrm{H}_{2} \mathrm{O}$ and $\mathrm{H}_{2} \mathrm{O}$ nanofluids

Journal of Applied Physics 109, 014914 (2011); https://doi.org/10.1063/1.3524274

Empirical correlation finding the role of temperature and particle size for nanofluid $\left(\mathrm{Al}_{2} \mathrm{O}_{3}\right)$ thermal conductivity enhancement

Applied Physics Letters 87, 153107 (2005); https://doi.org/10.1063/1.2093936

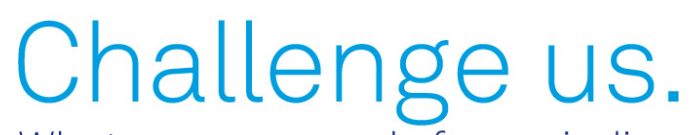

What are your needs for periodic

signal detection?

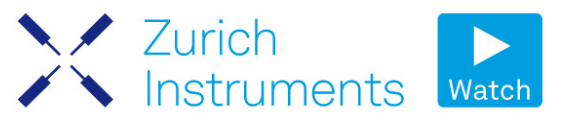

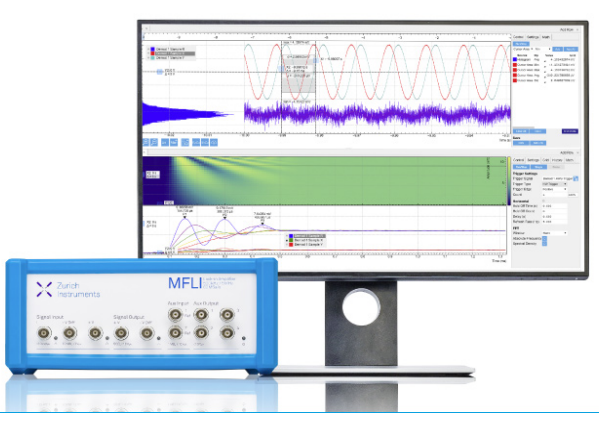

105, 064306 


\title{
An investigation of silicon carbide-water nanofluid for heat transfer applications
}

\author{
D. Singh, ${ }^{1, a)}$ E. Timofeeva ${ }^{2}$ W. Yu, ${ }^{2}$ J. Routbort, ${ }^{2}$ D. France, ${ }^{2}$ D. Smith, ${ }^{2}$ and \\ J. M. Lopez-Cepero ${ }^{3}$ \\ ${ }^{1}$ Nuclear Engineering Division, Argonne National Laboratory, Argonne, Illinois 60439, USA \\ ${ }^{2}$ Energy Systems Division, Argonne National Laboratory, Argonne, Illinois 60439, USA \\ ${ }^{3}$ University of Seville, Seville 41080, Spain
}

(Received 19 November 2008; accepted 10 January 2009; published online 18 March 2009)

\begin{abstract}
Thermal conductivity and mechanical effects of silicon carbide nanoparticles uniformly dispersed in water were investigated. Mean size of SiC particles was $170 \mathrm{~nm}$ with a polydispersity of $\sim 30 \%$ as determined from small-angle $\mathrm{x}$-ray scattering and dynamic light scattering techniques. Room temperature viscosity of the nanofluids ranged from 2 to $3 \mathrm{cP}$ for nominal nanoparticle loadings 4-7 vol \%. On a normalized basis with water, viscosity of the nanofluids did not significantly change with the test temperature up to $85^{\circ} \mathrm{C}$. Optical microscopy of diluted nanofluid showed no agglomeration of the nanoparticles. Thermal conductivity of the fluid was measured as a function of the nominal nanoparticle loading ranging from 1 to 7 vol \%. Enhancement in thermal conductivity was approximately $28 \%$ over that of water at 7 vol \% particle loadings under ambient conditions. Enhancements in thermal conductivities for the nanofluids with varying nanoparticle loadings were maintained at test temperatures up to $70{ }^{\circ} \mathrm{C}$. Results of thermal conductivity have been rationalized based on the existing theories of heat transfer in fluids. Implications of using this nanofluid for engineering cooling applications are discussed. (C) 2009 American Institute of Physics.
\end{abstract}

[DOI: 10.1063/1.3082094]

\section{INTRODUCTION}

Liquids with uniformly dispersed nanoparticles (nanofluids) have been extensively investigated for enhanced thermal properties. Over the last decade, there have been numerous publications in literature that reported enhanced thermal conductivity for fluids with few vol \% of nanoparticle additions. $^{1-5}$ Implications of nanofluids with enhanced thermal characteristics can be significant in terms of more efficient cooling systems, resulting in higher productivity and energy savings. Some potential applications for nanofluids could be for heat exchangers, radiators for engines, process cooling systems, microelectronics, etc.

Enhancements in the thermal conductivities for nanofluids, for the most part, follow the predictions of the effective medium theory (EMT) based on the Maxwell mean field theory initially developed for noninteracting spheres ${ }^{6}$ and extended for nonspherical particle shapes by Hamilton and Crosser. ${ }^{7}$ For low ( $<10$ vol \%) nanoparticle concentrations, Maxwell's theory predicts thermal conductivity enhancements as $k_{\mathrm{eff}} / k_{0}=1+3 \phi\left(k_{p}-k_{0}\right) /\left[2 k_{0}+k_{p}-\varphi\left(k_{p}-k_{0}\right)\right] \approx 1$ $+3 \phi$, where $k_{\text {eff }}, k_{p}$, and $k_{0}$ are thermal conductivities of nanofluid, solid particles, and the base fluid, respectively, and $\phi$ is the nanoparticle volume fraction. Modest thermal conductivity enhancements over Maxwell's predictions can be achieved by modifying the shape of the nanoparticles. ${ }^{8,9}$ Hamilton and Crosser ${ }^{7}$ modeled the range of enhancements based on the spherical to rod shapes of the particles. However, there are instances where the enhancements are significantly higher than EMT predictions at very low concentra-

\footnotetext{
${ }^{\text {a) }}$ Author to whom correspondence should be addressed. Electronic mail: dsingh@anl.gov.
}

tions of nanoparticles. ${ }^{1-4}$ These anomalous enhancements have typically been reported for metallic nanoparticles in fluids.

Thermal conduction enhancements in nanofluids have been attributed to variety of mechanisms, including Brownian motion, ${ }^{10}$ microconvection, ${ }^{11}$ clustering, and agglomeration. ${ }^{12,13}$ Literature provides no clear consensus on a specific mechanism; however, the general belief is that a combination of mechanisms may be operating and would be specific to a nanoparticle/fluid system and test conditions. Further, effect of nanoparticle interface layer on thermal conductivity is not clearly understood. A metal particle with surface oxidation would increase the interfacial resistance and consequently reduce the thermal conductivity. ${ }^{14}$

Unlike many metallic nanoparticles, ceramic nanoparticles are not susceptible to surface oxidation and are, thus, much easier to incorporate into a fluid. Further, their chemical stability over long period of times is significantly better than metals. Ceramics in general have low thermal conductivities with few exceptions such as carbides and nitrides. Silicon carbide has one of the highest bulk thermal conductivity $(\approx 120 \mathrm{~W} / \mathrm{m} \mathrm{K})$ among ceramics and is readily available. Further, recent theoretical modeling has shown that nanoparticles of wide band gap semiconductor material, such as $\mathrm{SiC}$, might be conducive to an additional radiative heat transfer mechanism in fluids. ${ }^{15}$ A limited study by Xie et al. ${ }^{8}$ on $\mathrm{SiC}$ nanoparticle based fluids showed thermal conductivity enhancements at $4{ }^{\circ} \mathrm{C}$. However, their study did not address thermal performance of the SiC nanofluid at elevated temperatures, and other mechanical properties of the fluid such as viscosity and fluid stability were not explored.

Industrial applications for nanofluid technology are in an embryonic stage. Potential for use of nanofluids for cooling 
systems such as radiators in trucks will require not only enhanced thermal properties but also minimal negative mechanical effects of the nanofluid in a closed system. In this regard, viscosity of the nanofluid is critical since it is a contributing factor to pumping power for the circulation of the nanofluid. Further, any erosive and clogging effects of the nanofluids on the fluid transmission lines or radiator can have an adverse effect on its usage.

The objective of this study was to investigate a commercially available $\mathrm{SiC} /$ water nanofluid. For this purpose, we conducted physical and microstructural characterizations of the nanofluid for particle size, viscosity, and agglomeration. Further, we investigated the effects of particle loadings and test temperature on the fluid viscosity and thermal conductivity. Results were used to correlate the physical properties to the thermal behavior of the nanofluid to assess the viability of the fluid for an industrial application.

\section{EXPERIMENTAL}

$\mathrm{SiC} /$ water nanofluid samples used for this investigation were obtained from Saint Gobain, Northboro, MA. Two batches were procured with nominal SiC loadings of 4 and 7.4 vol \%. The $\mathrm{pH}$ values of the fluids were maintained between 9 and 10, resulting in uniformly dispersed nanoparticles. No surfactants were added to the fluids. The asfabricated nanofluids showed no particle settling. For physical and thermal characterizations, as-received fluids were diluted to various (1-2 vol \%) nanoparticle loadings using de-ionized water adjusted to a $\mathrm{pH}$ of 10 with sodium hydroxide.

Optical and electron microscopic techniques were employed to look at the nanoparticles in water as well as after the water was evaporated. A special chamber was designed to hold a small volume of nanofluid (diluted by $\sim 100 X$ ) on an optical microscope. Dynamic and static images were recorded. Dried nanofluid powders were observed on a field emission scanning electron microscope (SEM) (Hitachi Model S-4700-II, Tokyo, Japan) to establish the shape and contour of the individual nanoparticles.

Particle size characterization of the nanofluids was conducted using two approaches: dynamic light scattering (DLS) and small-angle $\mathrm{x}$-ray scattering (SAXS). DLS was conducted using a table-top instrument (Brookhaven Instruments Corp., Holtsville, NY). SAXS on nanofluids was performed on beamline 15-ID-D at the Advanced Photon Source, Argonne National Laboratory, and the associated experimental details can be found elsewhere. ${ }^{16}$ Diluted nanofluids, to avoid particle-particle interactions, were exposed to monochromatic x-ray beam $(\lambda=1.5 \AA)$ and the scattered x-rays were collected on a detector. Information about the nanoparticles is derived from the plot of x-ray scattering intensity versus scattering momentum change $(q=4 \pi \sin \theta / \lambda)$, where $\theta$ is the scattering angle and $\lambda$ is the wavelength. Runs were also performed using the base fluid only and intensity signals from the nanofluids were appropriately corrected.

A Brookfield rotational type viscometer (Viscometer model DV-II+Pro, Middleboro, MA) was used to measure the viscosity of nanofluid samples. The viscometer consists

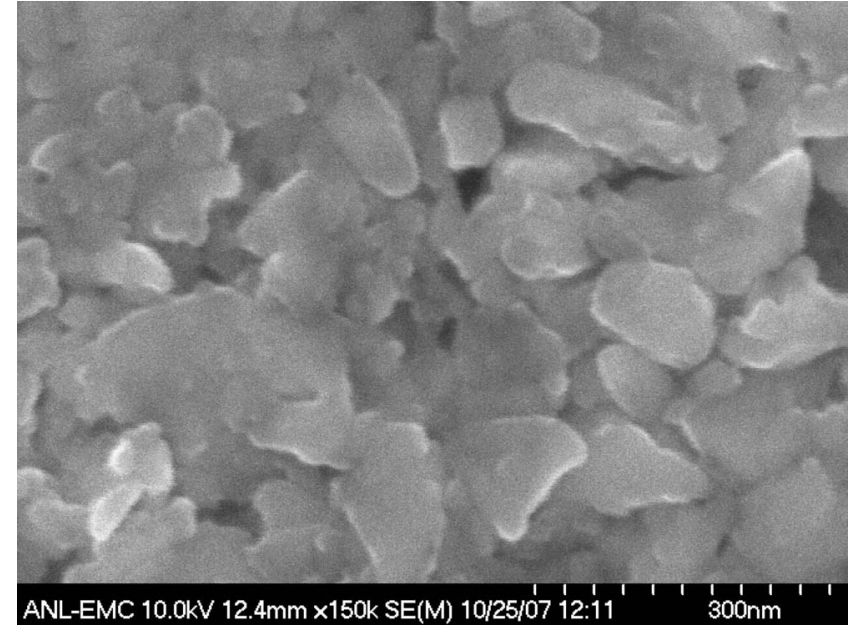

FIG. 1. Scanning electron micrograph of the SiC nanoparticles post drying the nanofluid.

of a stationary outer cylinder of radius $0.95 \mathrm{~cm}$ and inner cylinder of radius $0.87 \mathrm{~cm}$. Tests required about $8 \mathrm{ml}$ of nanofluid. Spindle SC4-18 was used in this study. The viscosities of nanofluids were measured at various shear rates ranging between 15 and $200 \mathrm{rpm}$. The temperature controller connected to the outer cylinder (temperature accuracy of $\pm 0.3{ }^{\circ} \mathrm{C}$ ) was used to control the temperature of the nanofluid samples from $15^{\circ} \mathrm{C}$ up to $55^{\circ} \mathrm{C}$ in increments of $10{ }^{\circ} \mathrm{C}$.

Transient hot-wire technique ${ }^{17}$ has been extensively used in the literature for thermal conductivity measurements for nanofluids and was applied in this study. Measurements were made on fluids with particle loadings ranging from 1 to 7.4 vol \%. In addition, test temperature was varied from ambient to $70{ }^{\circ} \mathrm{C}$ for fluids with approximately $1-4$ vol \% nanoparticle loadings. Elevated temperature thermal conductivity measurements were conducted by placing the entire hot-wire setup inside a temperature oven. Prior to the measurement, temperature of the oven was equilibrated for 30 min. Further, base line thermal conductivities of base water were determined at each test condition to establish the effect of particle additions.

\section{RESULTS}

Figure 1 shows the SEM image of the SiC nanoparticles obtained after drying out the water. As seen in the image, the particle size varies over a wide range from 60 to $250 \mathrm{~nm}$ in the longest direction. Both rounded as well as angular platelet like particles are observed. Figure 2 shows the distribution of $\mathrm{SiC}$ nanoparticles in the diluted fluid obtained using an optical microscope. The image shows no visible agglomeration of the nanoparticles; size of the dark spots on the image does not exceed $0.2 \pm 0.05 \mu \mathrm{m}$.

Results of particle size measurements using DLS technique are shown in Fig. 3. High polydispersity $(\sim 30 \%)$ of nanoparticles, observed from SEM and optical images, is also reflected in DLS spectra. Intensity and number distribution spectra show two peaks at 31 and $179 \mathrm{~nm}$. Average particle size determined by lognormal distribution was about $130 \mathrm{~nm}$. 


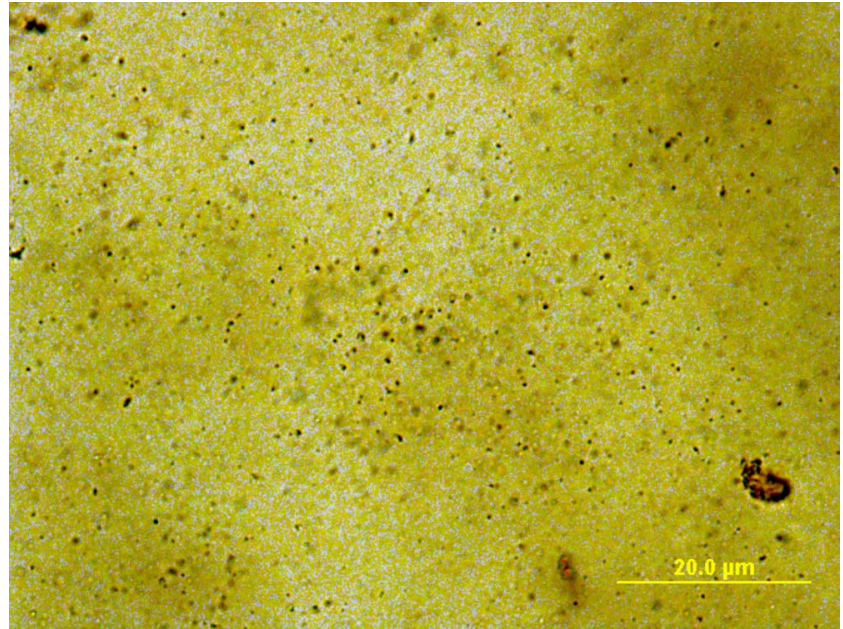

FIG. 2. (Color online) Optical micrograph of diluted SiC/water nanofluid showing dispersed $\mathrm{SiC}$ nanoparticles.

Figure 4 shows the one-dimensional SAXS pattern of diluted $\mathrm{SiC} /$ water nanofluid. The plot of $\mathrm{x}$-ray scattering intensity versus scattering momentum change $(Q$ $=4 \pi \sin \theta / \lambda)$ on a $\log -\log$ scale provides information about the nanoparticles. Because of large $(\sim 30 \%)$ polydispersity in the $\mathrm{SiC}$ particle size, there were no oscillations in the scattering intensity observed that normally arise from the inherent form-factor scattering from the particles that have a narrow distribution in sizes. Experimental scattering data were fitted using the standard maximum entropy mode ${ }^{18}$ that resulted to an average particle size of $170 \mathrm{~nm}$. The model assumes that the particles are spherical in shape.

Viscosities of the nanofluids $(1.8,3.7$, and 7.4 vol \% particle loadings) were measured as a function of temperature ranging from $15{ }^{\circ} \mathrm{C}$ to $55^{\circ} \mathrm{C}$ and compared to viscosity of the plain de-ionized water. Figure 5 shows the room temperature viscosity increase in the nanofluid with nanoparticle loadings. Viscosity variations for the nanofluids as a function of temperature are shown in Fig. 6. The viscosity of water decreases with increasing temperature from ambient value of $1.1 \mathrm{cP}$ at $15{ }^{\circ} \mathrm{C}$ to $0.5 \mathrm{cP}$ at $55^{\circ} \mathrm{C}$. With the addition of nanoparticles, fluid viscosity increases with nanoparticle loadings. The highest viscosity $(2.94 \mathrm{cP})$ was observed at $15^{\circ} \mathrm{C}$ for the nanofluid with 7.4 vol \% loading. With in-

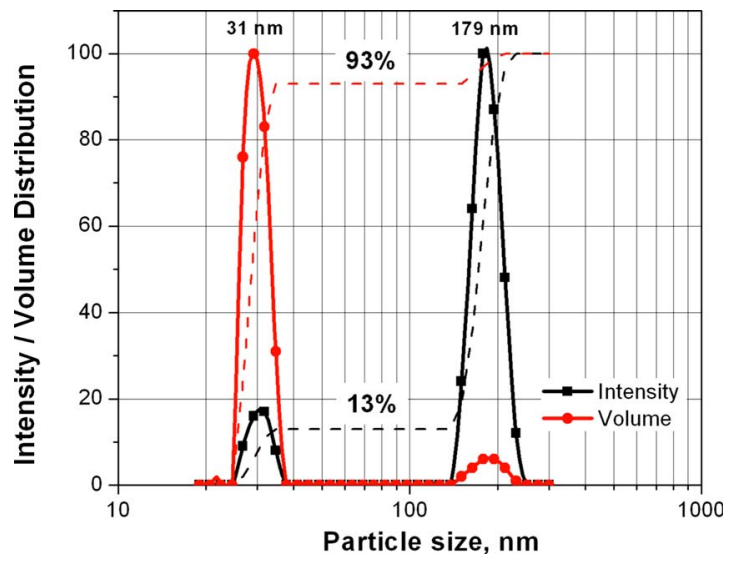

FIG. 3. (Color online) DLS results on the SiC/water nanofluid. creasing test temperatures, viscosities of the nanofluids also decreased similar to the viscosity of water. Due to limitation of the viscometer and the spindle, we were only getting reliable data in temperature range of $15-55^{\circ} \mathrm{C}$. Experimental data on nanofluids viscosity (Fig. 6) were fitted with the Arrhenius functional form ${ }^{19}$ to get an estimation of nanofluid's viscosity at higher temperatures,

$$
\eta=\eta_{\infty, T} e^{\left(E_{a} / R T\right)},
$$

where $\eta$ and $\eta_{\infty, T}$ are the measured viscosity and viscosity at infinite temperature, respectively, $E_{a}$ is the activation energy to viscous flow $(\mathrm{J} / \mathrm{mol}), R$ is the gas constant, and $T$ is the absolute temperature in $\mathrm{K}$.

Figure 7 presents the viscosities of the SiC-water nanofluids with three particle loadings normalized with that of base water as a function of test temperature. It is interesting to note that within the range of experimentally studied temperature, the normalized viscosities of $\mathrm{SiC}$ /water nanofluids are nearly constant.

Figure 8 shows the enhancement in thermal conductivity of the $\mathrm{SiC} /$ water nanofluid over water as a function of nanoparticle volume fraction. Thermal conductivity of the base water fluid $(\mathrm{pH}=10)$ was measured as $0.609(\mathrm{~W} / \mathrm{m} \mathrm{K})$ at ambient room temperature. Enhancements of as high as $28 \%$ are observed for a particle loading of $\approx 7.4 \mathrm{vol} \%$. Also shown in Fig. 8 is the prediction based on Maxwell's equation. At lower volume fractions, enhancements are quite similar to the prediction; however, at higher particle loadings $(>4 \mathrm{vol} \%)$, enhancements are higher than the prediction.

Thermal conductivity of nanofluids was measured at 23, 50 , and $70{ }^{\circ} \mathrm{C}$. Figure 9 shows the normalized enhancements in the thermal conductivity for the nanofluids as a function of temperature. The variation in the thermal conductivity of the base water fluid was measured and was used for normalization. For all the nanoparticle loadings investigated, enhancement in the thermal conductivity over that of water, at the specific test temperature, was maintained constant at all studied temperatures.

\section{DISCUSSION}

The silicon carbide-water nanofluid system exhibited an increase in the thermal conductivity as a function of particle loading at ambient room temperature. This result is consistent with observation on $\mathrm{SiC}$-water nanofluid system as reported by Xie et al. ${ }^{8}$ Interestingly, the enhancements over the Maxwell theory predictions for spherical particles are larger at higher particle loadings. This positive deviation can be rationalized based on the shape and/or aggregation effects of $\mathrm{SiC}$ nanoparticles at higher loadings. Hamilton-Crosser model $^{7}$ allows calculation of the thermal conductivity of two-component heterogeneous mixtures as a function of the conductivity of pure materials, composition of the mixture, and the manner in which the pure materials are distributed throughout the mixture [discontinuous phase (particles of various shapes) dispersed within continuous phase (base fluid) in either regular or irregular array]. The effective ( $\left.k_{\text {eff }}\right)$ conductivity enhancement is given as ${ }^{9}$ 


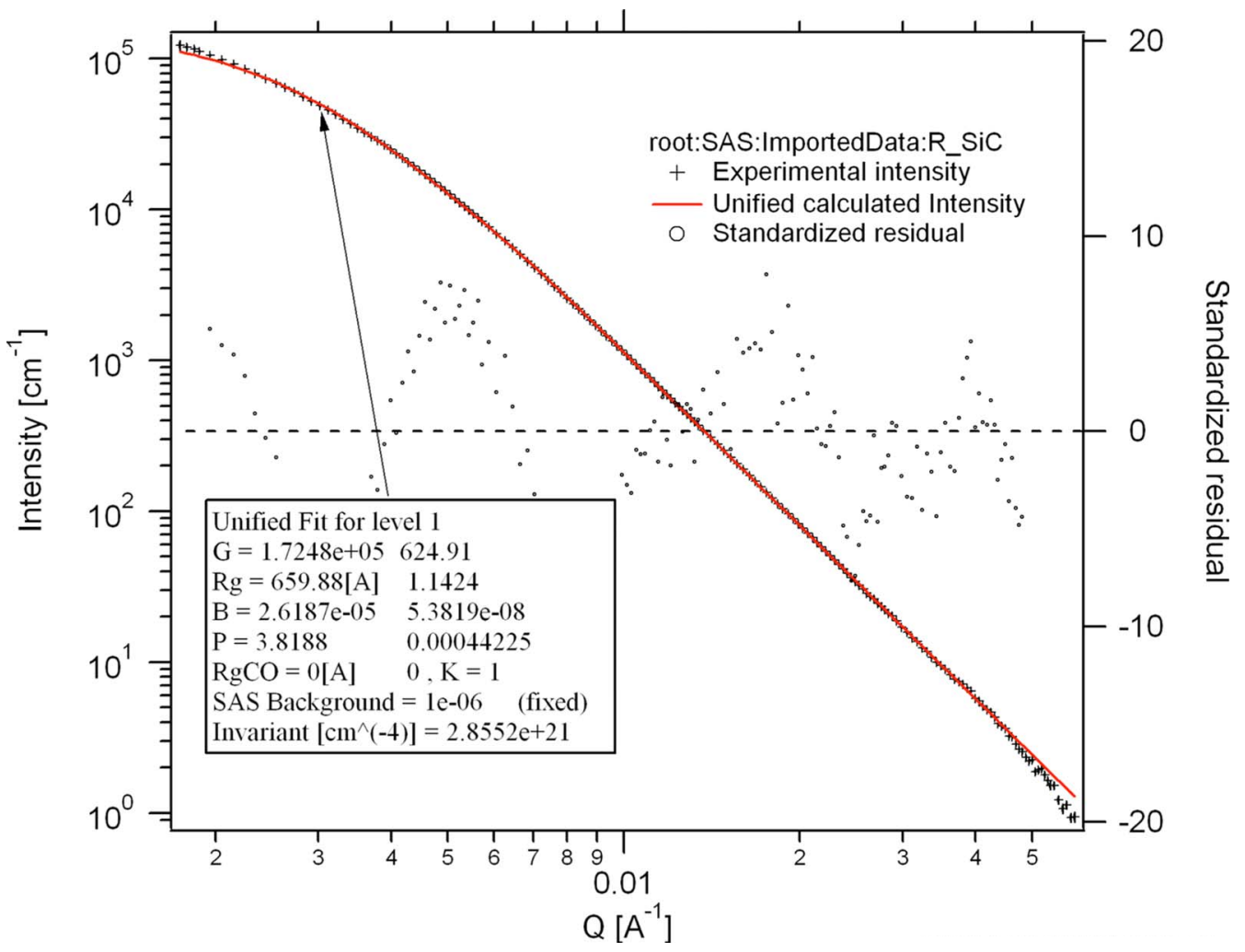

FIG. 4. (Color online) SAXS data of a diluted SiC nanofluid and a fit using the maximum entropy method assuming spherical particles with an average diameter of $170 \mathrm{~nm}$. Dotted points are standardized residuals.

$$
\frac{k_{\mathrm{eff}}}{k_{0}}=\frac{k_{p}+(n-1) k_{0}+(n-1)\left(k_{p}-k_{0}\right) \phi}{k_{p}+(n-1) k_{0}-\left(k_{p}-k_{0}\right) \phi},
$$

where $k_{p}$ and $k_{0}$ are the conductivities of the particle material and the base fluid, respectively, $\phi$ is the particle volume fraction, $n$ is the empirical shape factor given by $n=3 / \psi$, and $\psi$ is the sphericity defined as the ratio of the surface area of the particle and the equivalent surface area of the sphere for a constant volume. For spherical shaped particles, the sphericity is 1 and it corresponds to a shape factor of 3 for which Hamilton-Crosser equation reduces to Maxwell's equation for the conductivity of randomly distributed and noninteracting homogeneous spheres in a continuous medium. Further,

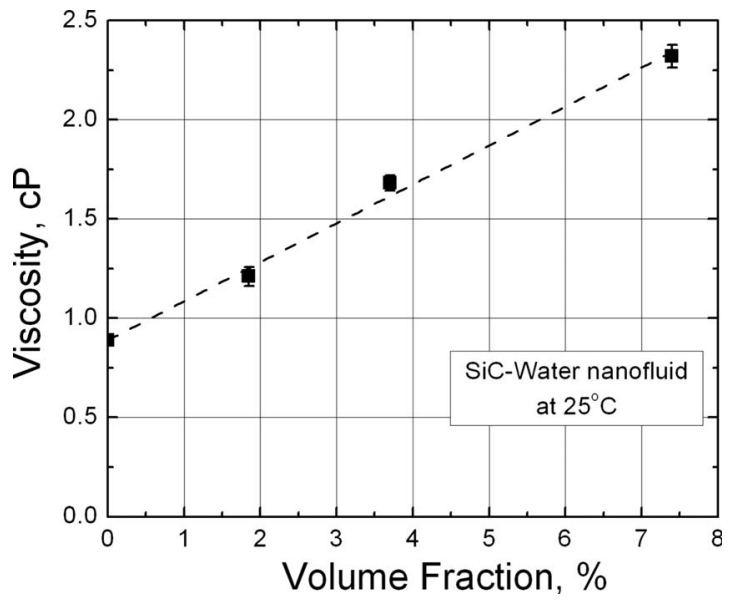

FIG. 5. Viscosity of SiC/water nanofluid as a function of SiC particle volume fraction. $n=6$ corresponds to cylindrical shaped particles with $1: 17$ aspect ratio.

Figure 10 shows the thermal conductivity enhancements for $\mathrm{SiC}$-water system bounded by the predictions (solid lines) of the Hamilton-Crosser model for $n=3$ and $n=6$. Dashed line is the prediction based on the shape factor of 4.2 and agrees well with the experimental data. Several particle shapes are possible for the same value of shape factor. However, as seen in the SEM image (Fig. 1), shapes of SiC nanoparticles are better described as disks or platelets. Ratio of diameter to height of the disk like particles can be estimated from the shape factor for the investigated $\mathrm{SiC}$-water nanofluid and is found to be $\approx 4: 1$.

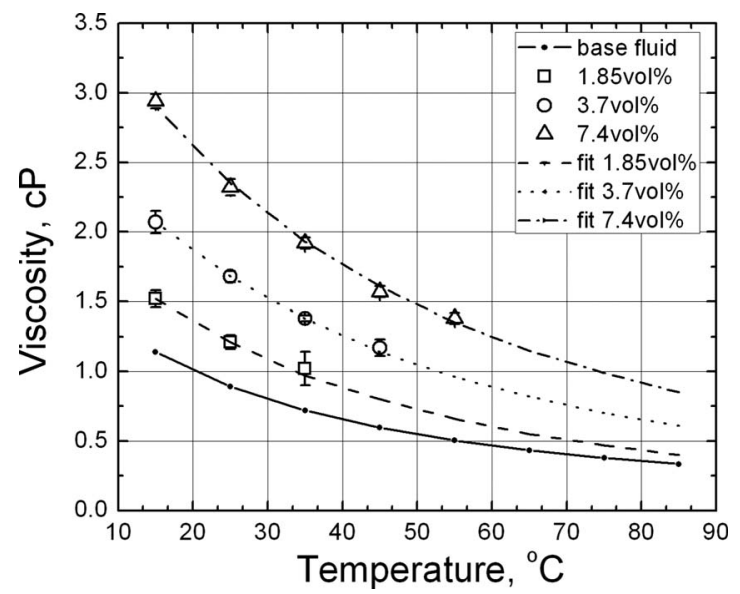

FIG. 6. Viscosity of SiC/water nanofluids with varying particle loadings as a function of temperature. 


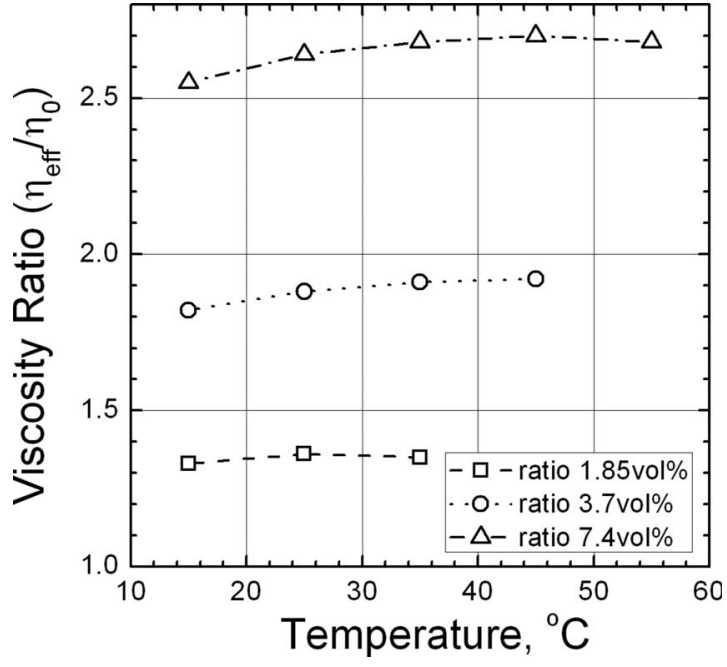

FIG. 7. Viscosity SiC/water nanofluids with varying particle loadings normalized with respect to the viscosity of water at room temperature.

Enhancements in thermal conductivity at higher particle loadings can be due to the contribution from the shapes of individual $\mathrm{SiC}$ nanoparticles or the formation of aggregates or clusters of $\mathrm{SiC}$ particles ${ }^{9}$ or a combination of both. Even though separated particles were observed with the optical microscopy conducted on highly diluted suspensions, one cannot exclude formation of agglomerates at higher volume fractions of nanoparticles. To ensure the possible effects of the particle shapes in situ high-resolution microscopy of the nanofluid will be required.

General trends in thermal conductivity enhancement of $\mathrm{SiC} /$ water nanofluid can be explained by EMT using the Hamilton-Crosser modification for nonspherical shape factors. In the present study, thermal conductivity enhancements, relative to the base fluid at a specific temperature, showed temperature independence for the three different nanoparticle loadings (Fig. 9). This observation is consistent with EMT, i.e., no temperature dependence of the thermal conductivity enhancement is considered by this model. These observations also agree with other experimental studies. ${ }^{9,20}$

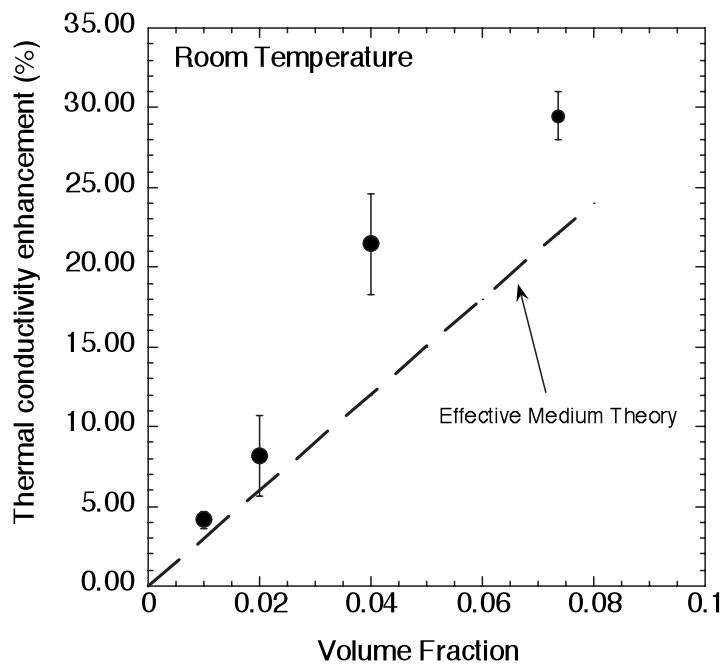

FIG. 8. Plot of the thermal conductivity enhancement as a function of $\mathrm{SiC}$ nanoparticle loading at room temperature.

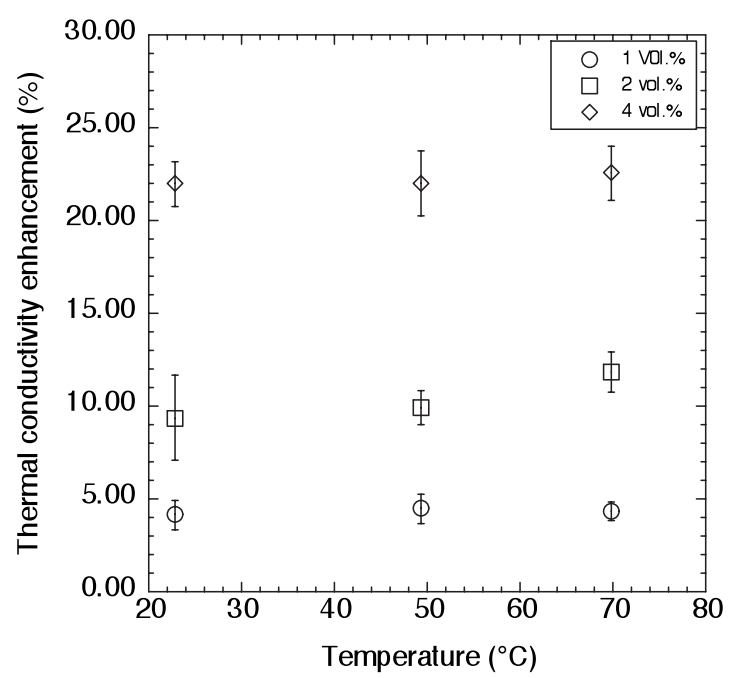

FIG. 9. Thermal conductivity enhancements for SiC/water nanofluids as a function of temperature for varying nanoparticle loadings.

However, increase in thermal conductivity with temperature for nanofluids have been reported in the literature (Ref. 21 and references therein). These increases in thermal conductivity have been rationalized on the basis of Brownian motion as the mechanism for heat conduction, which would be temperature dependent ${ }^{22}$ and specific to the nanofluid system. Nevertheless, maintaining thermal conductivity enhancements at elevated temperatures is desirable for elevated temperature thermal management.

Measured viscosity for $\mathrm{SiC}$-water nanofluids are relatively low and linearly increase with the concentration of nanoparticles (Fig. 5). Further, there was no shear rate dependence of the viscosity indicative of a Newtonian fluid behavior. Applying the Arrhenius relation to the experimental data yielded activation energy for the $\mathrm{SiC}$-water system to be $14-16 \mathrm{~kJ} / \mathrm{mol}$. These values were somewhat lower than for pure water $(16.45 \mathrm{~kJ} / \mathrm{mol})$. For the particle loadings in-

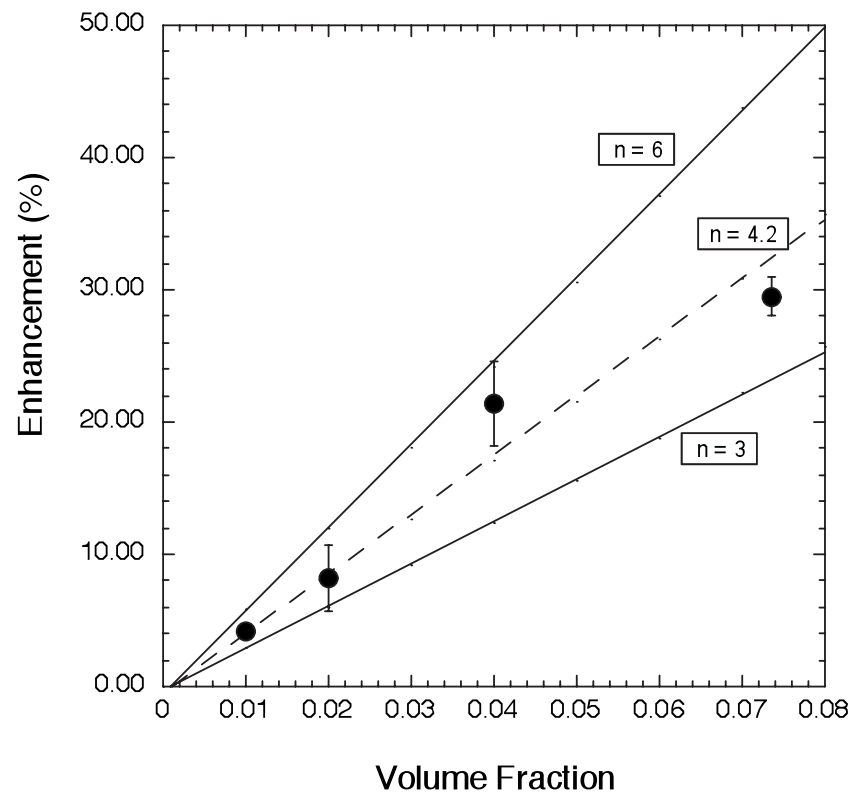

FIG. 10. Comparison of the observed enhancements of thermal conductivity with the predictions from the Hamilton-Crosser model. 
vestigated, there was no significant dependence of activation energy on particle loading. Physical interpretation of activation energy of viscous flow is not developed well for nanoparticle suspension but described as the mean potential energy of interaction of two molecules for pure liquids ${ }^{23}$ or as the steepness of the temperature dependence of ceramic suspension viscosity. ${ }^{24}$ Thus, it appears that addition of $\mathrm{SiC}$ nanoparticles to water slightly decreases activation energy of viscous flow, and at increasing temperatures viscosity of nanofluids will decrease faster than viscosity of the base fluid. However, the studied temperature interval was too small to see this effect.

Lower viscosity implies lower pumping power that is advantageous from an industrial application standpoint. Prasher et al $^{25}$ derived a quantitative expression for fully developed laminar flow that compares the relative coefficients of viscosity and thermal conductivity enhancements to predict the potential of the nanofluid in an actual application. As observed in our experiments, both viscosity and thermal conductivity enhancements at low particle volume fractions can be described by linear dependence on the particle volume fraction $(\phi)$,

$$
\frac{k_{\mathrm{eff}}}{k_{0}}=1+C_{k} \phi \quad \text { and } \quad \frac{\eta_{\mathrm{eff}}}{\eta_{0}}=1+C_{\eta} \phi,
$$

where $C_{\eta}$ and $C_{k}$ are the viscosity and thermal conductivity enhancement coefficients. At low volume fractions, for the nanofluid to be beneficial, the ratio of coefficients $\left(C_{\eta} / C_{k}\right)$ should be $<4 .{ }^{25}$ Estimated room temperature average values for $C_{\eta}$ and $C_{k}$ were 21 and 4.2, respectively. Thus, the ratio, $C_{\eta} / C_{k}$, was slightly higher than expected for efficient nanofluid use, implying that either viscosity needs to be reduced (viscosity modifiers) or conversely the thermal conductivity at the same particle loading has to be improved. However, it should be noted that the Prasher model ${ }^{25}$ was developed for laminar flow. In turbulent regimes where potentially water based nanofluids would be used, the Mouromtseff number (Mo) can be used to compare two fluids, ${ }^{26}$

$$
\mathrm{Mo}=\frac{\rho^{0.8} C_{p}^{0.4} k^{0.6}}{\mu^{0.4}},
$$

where $k, \rho, C_{p}$, and $\mu$ are the thermal conductivity, density, specific heat, and viscosity, respectively. The fluid with the higher Mo provides a larger heat transfer coefficient at the same velocity for a particular system. Mo ratio of SiC-water nanofluids to water ranges from 0.95 to 0.83 for particle concentrations from $1.85 \%$ to $7.4 \%$. Thus, in its current state the SiC-water nanofluid may not be as good as water for heat transfer in the turbulent regime. However, Mo does not incorporate any additional heat transfer mechanisms that have been observed in nanofluid heat transfer studies, ${ }^{27}$ and therefore, experiments need to be conducted before conclusions can be drawn of the fluid potential.

\section{CONCLUSIONS}

A commercial SiC-water based nanofluid has been investigated for thermal conductivity and viscosity character- istics. Thermal conductivity increases with particle loadings and is higher than the predictions of EMT for noninteracting spheres. These enhancements can be explained based on the $\mathrm{SiC}$ nanoparticle shapes and/or clusters. Viscosity also increases with addition of nanoparticles. Effects of temperature on viscosity and thermal conductivity were established. Based on the conductivity/viscosity data, preliminary estimation of the potential of this nanofluid system has been made.

\section{ACKNOWLEDGMENTS}

This work was sponsored by Michelin American Research and Development Corporation and by the U.S. Department of Energy under Contract No. DE-AC0206CH11357 at Argonne National Laboratory, managed by the University of Chicago Argonne LLC (USA). Use of the Advanced Photon Source was supported by the Office of Science, Office of Basic Energy Sciences, U.S. Department of Energy under Contract No. DE-AC02-06CH11357. Assistance from Dr. D. J. Cookson in performing SAXS run is highly appreciated. Authors are grateful to Dr. Steve Hartline of Saint Gobain for providing the SiC-water nanofluid formulations for this study.

${ }^{1}$ T. Cho, I. Baek, J. Lee, and S. Park, J. Ind. Eng. Chem. (Seoul, Repub. Korea) 11, 400 (2005)

${ }^{2}$ T. K. Hong, H. S. Yang, and C. J. Choi, J. Appl. Phys. 97, 064311 (2005). ${ }^{3}$ J. A. Eastman, S. U. S. Choi, S. Li, W. Yu, and L. J. Thompson, Appl. Phys. Lett. 78, 718 (2001).

${ }^{4}$ M. Chopkar, S. Kumar, D. R. Bhandari, P. K. Das, and I. Manna, Mater. Sci. Eng., B 139, 141 (2007).

${ }^{5}$ H. U. Kang, S. H. Kim, and J. M. Oh, Exp. Heat Transfer 19, 181 (2006). ${ }^{6}$ J. C. Maxwell, A Treatise on Electricity and Magnetism, 2nd ed. (Clarendon, Oxford, 1881).

${ }^{7}$ R. L. Hamilton and O. K. Crosser, IEICE Trans. Fundamentals 1, 187 (1962).

${ }^{8}$ H. Xie, J. Wang, T. Xi, Y. Liu, and F. Ai, J. Mater. Sci. 21, 193 (2002).

${ }^{9}$ E. Timofeeva, A. N. Gavrilov, J. M. McCloskey, Y. V. Tolmachev, S. Sprunt, L. M. Lopatina, and J. V. Selinger, Phys. Rev. E 76, 061203 (2007).

${ }^{10}$ S. P. Jang and S. U. S. Choi, Appl. Phys. Lett. 84, 4316 (2004).

${ }^{11}$ R. Prasher, P. Bhattacharya, and P. E. Phelan, ASME J. Heat Transfer 128, 588 (2006)

${ }^{12}$ R. Prasher, P. E. Phelan, and P. Bhattacharya, Nano Lett. 6, 1529 (2006).

${ }^{13}$ B.-X. Wang, L.-P. Zhou, and X.-F. Peng, Int. J. Heat Mass Transfer 46, 2665 (2003).

${ }^{14}$ J. A. Eastman, S. R. Phillpot, S. U. S. Choi, and P. Keblinski, Annu. Rev. Mater. Res. 34, 219 (2004).

${ }^{15} \mathrm{~T}$-W. Tam (unpublished).

${ }^{16}$ D. J. Cookson, N. Kirby, R. Knott, M. Lee, and D. Schultz, J. Synchrotron Radiat. 13, 440 (2006)

${ }^{17}$ W. Yu and S. U. S. Choi, Rev. Sci. Instrum. 77, 076102 (2006).

${ }^{18}$ J. A. Potton, G. J. Daniell, and B. D. Rainford, J. Appl. Crystallogr. 21, 663 (1988).

${ }^{19}$ R. G. Larson, The Structure and Rheology of Complex Fluids (Oxford University Press, New York, 1999).

${ }^{20}$ D. C. Venerus, M. S. Kabadi, S. Lee, and V. Perez-Luna, J. Appl. Phys. 100, 094310 (2006).

${ }^{21}$ W. Yu, D. M. France, S. U. S. Choi, and J. L. Routbort, http:// www.transportation.anl.gov/pdfs/MM/401.pdf, 2007.

${ }^{22}$ S. K. Das, N. Putra, P. Thiesen, and W. Roetzel, ASME J. Heat Transfer 125, 567 (2003).

${ }^{23}$ T. H. S. Higgins and C. J. G. Raw, J. Chem. Phys. 27, 623 (1957).

${ }^{24} \mathrm{M}$. Trunec and J. Hrazdera, Ceram. Int. 31, 845 (2005).

${ }^{25}$ R. Prasher, D. Song, and J. Wang, Appl. Phys. Lett. 89, 133108 (2006).

${ }^{26}$ I. E. Mouromtseff, Proc. IRE 30, 190 (1942).

${ }^{27}$ Y. Xuan and Q. Li, ASME J. Heat Transfer 125, 151 (2003). 